\title{
Multiscale Asymptotic Analysis and Parallel Algorithm of Parabolic Equation in Composite Materials
}

\author{
Xin Wang, Xi-liang Duan, and Yang Gao \\ Department of Mathematics, Shanghai University, Shanghai 200444, China \\ Correspondence should be addressed to Xin Wang; xinwang@shu.edu.cn \\ Received 19 September 2013; Accepted 3 March 2014; Published 8 April 2014 \\ Academic Editor: Igor Andrianov
}

Copyright (C) 2014 Xin Wang et al. This is an open access article distributed under the Creative Commons Attribution License, which permits unrestricted use, distribution, and reproduction in any medium, provided the original work is properly cited.

An efficient parallel multiscale numerical algorithm is proposed for a parabolic equation with rapidly oscillating coefficients representing heat conduction in composite material with periodic configuration. Instead of following the classical multiscale asymptotic expansion method, the Fourier transform in time is first applied to obtain a set of complex-valued elliptic problems in frequency domain. The multiscale asymptotic analysis is presented and multiscale asymptotic solutions are obtained in frequency domain which can be solved in parallel essentially without data communications. The inverse Fourier transform will then recover the approximate solution in time domain. Convergence result is established. Finally, a novel parallel multiscale FEM algorithm is proposed and some numerical examples are reported.

\section{Introduction}

Consider the parabolic equation with rapidly oscillating coefficients as follows:

$$
\begin{gathered}
\frac{\partial u^{\varepsilon}(x, t)}{\partial t}-\frac{\partial}{\partial x_{i}}\left(a_{i j}\left(\frac{x}{\varepsilon}\right) \frac{\partial u^{\varepsilon}(x, t)}{\partial x_{j}}\right)=f(x, t), \\
(x, t) \in \Omega \times(0, T), \\
u^{\varepsilon}(x, t)=0, \quad(x, t) \in \partial \Omega \times(0, T), \\
u^{\varepsilon}(x, 0)=\bar{u}(x), \quad x \in \Omega,
\end{gathered}
$$

where $\Omega \in \mathscr{R}^{n}$ is a bounded smooth domain or a bounded polygonal convex domain with a periodic microstructure and $(0, T)$ denotes the time domain. The unknown is the temperature increment $u^{\varepsilon}(x, t) . f(x, t)$ and $\bar{u}(x)$ are some given functions which denote heat source and initial temperature, respectively. $\varepsilon>0$ is a small parameter and it represents the relative size of a periodic cell.

Let $\xi=x / \varepsilon$ and the heat conductivity coefficient $\left(a_{i j}(\xi)\right)_{1 \leq i, j \leq n}$ is assumed to satisfy the following.

$\left(A_{1}\right) a_{i j}(x / \varepsilon)=a_{i j}(\xi)$ is $Q$-periodic in $\xi, \forall i, j=1, \ldots, n$, where $Q=(0,1)^{n}$ is the reference cell.
$\left(A_{2}\right)\left(a_{i j}(\xi)\right)_{1 \leq i, j \leq n}$ is symmetric and satisfies the uniform elliptic condition; that is,

$$
\begin{gathered}
a_{i j}(\xi)=a_{j i}(\xi), \quad \forall i, j=1, \ldots, n, \\
\lambda_{1}|\eta|^{2} \leq a_{i j}(\xi) \eta_{i} \eta_{j} \leq \lambda_{2}|\eta|^{2}, \quad \text { a.e. } \xi \in Q ;
\end{gathered}
$$

where is $\eta=\left(\eta_{1}, \ldots, \eta_{n}\right)$ an arbitrary vector in $\mathscr{R}^{n}$, and $\lambda_{1}, \lambda_{2}>0$ are some constants independent of $\varepsilon$.

$\left(A_{3}\right)$ Let $Q \subset \subset Q^{\prime}$ and $Q^{\prime}=\left(\bigcup_{m=1}^{L} \bar{D}_{m}\right) \backslash \partial Q^{\prime}$. Suppose that the boundaries $\partial D_{m} \in C^{1, \gamma}$ for some $0<\gamma<1$ and $a_{i j}(\xi) \in C^{\mu}\left(\bar{D}_{m}\right), \forall i, j=1, \ldots, n$ for some $0<\mu<1$; see $[1,2]$.

In (1), the rapid spatial oscillations in the coefficients translate the periodic structure of the body which comes from assembling $\varepsilon$-scaled versions of the reference cell $Q$. This body has to be thought of as being made of a composite material.

An efficient analysis approach to such a problem induces a need for homogenization. The mathematical theory has been presented in a lot of works; see, for example, [3-6]. Several efficient numerical methods have been proposed and analyzed; for instance, see [7-9]. From these literatures , we found that the homogenized equation happens to be 
a system of the same type with constant coefficients. So the normal step-by-step time-marching algorithms such as the backward-Euler, Crank-Nicolson methods, or any higher order methods need to be applied to solve the homogenized equation step by step in time. Although these algorithms are effective in solving many practical problems, one of major drawbacks is that they are not easily parallelizable along the time axis, since they require the information of the solutions at previous time steps in order to advance to the next time step.

In this work, in order to propose a parallelizable multiscale numerical algorithm, we apply the Fourier transform and inverse Fourier transform to present the multiscale asymptotic analysis for (1). Numerical methods for timedependent problems based on the use of Fourier transform have been considered in $[10,11]$, providing us with the starting point for using Fourier transform in multiscale asymptotic analysis. In [10, 11], Douglas et al. introduced an efficient parallel method for solving wave equations in the space-time domain after taking the Fourier transforms. The parallel method had been further developed and extended to viscoelasticity, parabolic problems, and Navier-Stokes equations; see $[12,13]$. Later Sheen et al. also applied Laplace transform to solve parabolic problems; see $[14,15]$. In addition, the early study of homogenization by employing means of integral transformation is [16], where Fourier and Laplace transforms were applied to prove the weak convergence theorems of the homogenization procedure for the timedependent equations.

The main procedures in this paper are now briefly described. First, we take the Fourier transform with respect to the time variable in the parabolic equation and then present the multiscale asymptotic expansions of the solutions for the resulting equations in space-frequency domain at specific frequency points of interest, which are independent and may therefore be done in parallel. Finally, the approximate solution of parabolic equation in space-time domain is retrieved by the discrete inverse Fourier transform. It should be stated that our purpose of using Fourier transform is not just to prove the homogenization convergence theorems as done in [16], but to propose a parallelizable multiscale numerical algorithm. The new contributions presented in this study are the following. The strong convergence theorem of the second-order multiscale asymptotic solution in spacefrequency domain is derived where we carefully estimate the bounds associated with frequency variable $\omega$ as it has a singularity for both zero and infinity in inverse Fourier transform. The convergence result for the approximate solution in time domain is obtained. A novel parallel multiscale FEM algorithm and numerical examples in $3 \mathrm{D}$ composite materials are given.

The remainder of this paper is organized as follows. In Section 2, the original parabolic equation is transformed to a set of complex-valued elliptic problems. The multiscale asymptotic expansions in space-frequency domain are then discussed. In Section 3, the multiscale truncation error estimates in space-frequency domain are derived under some assumptions. The approximate solution in space-time domain is inverted back by discrete inverse Fourier transform and the convergence result is proved in Section 4. Finally, a multiscale algorithm is proposed and numerical simulations are carried out to validate the presented method.

Throughout the paper, let $C$ denote a positive constant independent of $\varepsilon$ and $\omega$. All complex-valued functions are assumed to have values in the complex field $\mathscr{C}$ and standard notations for function spaces and their associated norms will be used in this paper.

\section{Fourier Transform and Multiscale Asymptotic Expansions}

Denote the Fourier transform of a function $\rho(\cdot, t)$ by $\widehat{\rho}(\cdot, \omega)$, where

$$
\widehat{\rho}(\cdot, \omega)=\int_{-\infty}^{+\infty} \rho(\cdot, t) e^{-i \omega t} d t .
$$

In order to take the Fourier transform of (1), we first extend $u^{\varepsilon}$ and $f$ by zero when $t<0$ and then we transfer (1) into the following complex-valued elliptic problem in spacefrequency domain,

$$
\begin{gathered}
i \omega \widehat{u}^{\varepsilon}(x, \omega)-\frac{\partial}{\partial x_{i}}\left(a_{i j}(\xi) \frac{\partial \widehat{u}^{\varepsilon}(x, \omega)}{\partial x_{j}}\right) \\
=\widehat{f}(x, \omega)+\bar{u}(x), \quad x \in \Omega, \\
\widehat{u}^{\varepsilon}(x, \omega)=0, \quad x \in \partial \Omega,
\end{gathered}
$$

where $\omega$ is some fixed parameter in frequency domain.

It is not difficult to check the existence and uniqueness of the problem (4) with an application of the Lax-Milgram lemma; see [17].

Proposition 1. Let $\omega>0$, under the assumptions $\left(A_{1}\right)-\left(A_{2}\right)$; then, for any $\hat{f}(\cdot, \omega), \bar{u} \in H^{-1}(\Omega)$, (4) has a unique weak solution $\widehat{u}^{\varepsilon}(\cdot, \omega)$.

The method requires solving a finite set of problem (4) at specific frequency point $\omega$. It is natural to treat them simultaneously because they are independent. Moreover, observe that (4) is an equation with rapidly oscillating coefficients in spatial domain. The direct accurate numerical computation of the solution is difficult especially in $3 \mathrm{D}$ problems because it would require huge computational cost. We will apply the method of multiscale asymptotic expansion. Let the derivative with respect to $x$ be

$$
\frac{\partial}{\partial x_{i}}=\frac{\partial}{\partial z_{i}}+\varepsilon^{-1} \frac{\partial}{\partial \xi_{i}}, \quad i=1, \ldots, n,
$$

where $z$ denotes the macroscopic variable and $\xi$ describes the microscopic one. Two scales describe the model: $z$ gives the position of a point in domain $\Omega$ while $\xi$ gives its position in the reference cell $Q$. On expanding $\widehat{u}^{\varepsilon}$ in asymptotic expansion of the form

$$
\widehat{u}^{\varepsilon}(x, \omega)=\sum_{i=0}^{\infty} \varepsilon^{i} \widehat{u}_{i}(z, \xi, \omega),
$$


where $\widehat{u}_{i}(z, \xi, \omega)$ is Q-periodic in $\xi$, the space-frequency (4) becomes

$$
\begin{aligned}
& -\varepsilon^{-2}\left[\frac{\partial}{\partial \xi_{i}}\left(a_{i j}(\xi) \frac{\partial \widehat{u}_{0}(z, \xi, \omega)}{\partial \xi_{j}}\right)\right] \\
& -\varepsilon^{-1}\left[\frac{\partial}{\partial \xi_{i}}\left(a_{i j}(\xi)\left(\frac{\partial \widehat{u}_{0}(z, \xi, \omega)}{\partial z_{j}}+\frac{\partial \widehat{u}_{1}(z, \xi, \omega)}{\partial \xi_{j}}\right)\right)\right. \\
& \left.+\frac{\partial}{\partial z_{i}}\left(a_{i j}(\xi) \frac{\partial \widehat{u}_{0}(z, \xi, \omega)}{\partial \xi_{j}}\right)\right]+i \omega \widehat{u}_{0}(z, \xi, \omega) \\
& -\frac{\partial}{\partial z_{i}}\left(a_{i j}(\xi)\left(\frac{\partial \widehat{u}_{0}(z, \xi, \omega)}{\partial z_{j}}+\frac{\partial \widehat{u}_{1}(z, \xi, \omega)}{\partial \xi}\right)\right) \\
& -\frac{\partial}{\partial \xi_{i}}\left(a_{i j}(\xi)\left(\frac{\partial \widehat{u}_{1}(z, \xi, \omega)}{\partial z_{j}}+\frac{\partial \widehat{u}_{2}(z, \xi, \omega)}{\partial \xi_{j}}\right)\right)+O\left(\varepsilon^{1}\right) \\
& =\hat{f}(z, \omega)+\bar{u}(z), \quad(z, \xi) \in \Omega \times R^{n}, \\
& \sum_{i=0}^{\infty} \varepsilon^{i} \widehat{u}_{i}(z, \xi, \omega)=0, \quad(z, \xi) \in \partial \Omega \times R^{n}, \\
& \widehat{u}_{i}(z, \xi, \omega) \quad Q-\operatorname{periodic} \text { in } \xi .
\end{aligned}
$$

On equating orders at $O\left(\varepsilon^{-2}\right)$, with the well-known theorem of the existence and uniqueness of the solutions in quotient space, we deduce that

$$
\widehat{u}_{0}(z, \xi, \omega)=\widehat{u}_{0}(z, \omega) .
$$

Equating terms at $O\left(\varepsilon^{-1}\right)$, we have

$$
\begin{aligned}
&-\frac{\partial}{\partial \xi_{i}}\left(a_{i j}(\xi) \frac{\partial \widehat{u}_{1}(z, \xi, \omega)}{\partial \xi_{j}}\right) \\
&=\frac{\partial a_{i j}(\xi)}{\partial \xi_{i}} \frac{\partial \widehat{u}_{0}(z, \omega)}{\partial z_{j}}, \quad(z, \xi) \in \Omega \times R^{n} \\
& \widehat{u}_{1}(z, \xi, \omega) \quad \text { Q-periodic in } \xi
\end{aligned}
$$

with the method of separation of variables and we can obtain

$$
\widehat{u}_{1}(z, \xi, \omega)=N_{k}(\xi) \frac{\partial \widehat{u}_{0}(z, \omega)}{\partial z_{k}},
$$

where $N_{k}(\xi)(k=1, \ldots, n)$ are the solutions of the following problems:

$$
\begin{aligned}
-\frac{\partial}{\partial \xi_{i}}\left(a_{i j}(\xi) \frac{\partial N_{k}(\xi)}{\partial \xi_{j}}\right) & =\frac{\partial a_{i k}(\xi)}{\partial \xi_{i}}, \quad \xi \in R^{n} \\
& N_{k}(\xi) \quad \text { Q-periodic. }
\end{aligned}
$$

At $\left(\varepsilon^{0}\right)$, we have

$$
\begin{gathered}
i \omega \widehat{u}_{0}(z, \omega)-a_{i j}(\xi) \frac{\partial^{2} \widehat{u}_{0}(z, \omega)}{\partial z_{i} \partial z_{j}}-a_{i j}(\xi) \frac{\partial N_{k}(\xi)}{\partial \xi_{j}} \frac{\partial^{2} \widehat{u}_{0}(z, \omega)}{\partial z_{i} \partial z_{k}} \\
-\frac{\partial\left(a_{i j}(\xi) N_{k}(\xi)\right)}{\partial \xi_{i}} \frac{\partial^{2} \widehat{u}_{0}(z, \omega)}{\partial z_{j} \partial z_{k}} \\
-\frac{\partial}{\partial \xi_{i}}\left(a_{i j}(\xi) \frac{\partial \widehat{u}_{2}(z, \xi, \omega)}{\partial \xi_{j}}\right) \\
=\widehat{f}(z, \omega)+\bar{u}(z), \quad(z, \xi) \in \Omega \times R^{n}, \\
\widehat{u}_{2}(z, \xi, \omega) \quad Q-\text { periodic in } \xi .
\end{gathered}
$$

On averaging (12) over $Q$, we get the homogenized equation

$$
\begin{gathered}
i \omega \widehat{u}_{0}(z, \omega)-\frac{\partial}{\partial z_{i}}\left(\widehat{a}_{i j} \frac{\partial \widehat{u}_{0}(z, \omega)}{\partial z_{j}}\right) \\
=\widehat{f}(z, \omega)+\bar{u}(z), \quad z \in \Omega, \\
\widehat{u}_{0}(z, \omega)=0, \quad z \in \partial \Omega,
\end{gathered}
$$

where the homogenized coefficients are defined by

$$
\widehat{a}_{i j}=\frac{1}{|Q|} \int_{Q} a_{i j}(\xi)+a_{i k}(\xi) \frac{\partial N_{j}(\xi)}{\partial \xi_{k}} d \xi .
$$

Remark 2. Under the assumptions $\left(A_{1}\right)-\left(A_{2}\right)$ of the coefficients for the parabolic equation, one can prove that the homogenized coefficients $\widehat{a}_{i j}$ are also symmetric and uniform elliptic for some constants $\gamma_{1}, \gamma_{2}>0$; see, for example, [3, 5]. Therefore the existence and uniqueness of the solution $\widehat{u}_{0}$ for the homogenized (13) can be established.

Subtracting (13) from (12), we have

$$
\begin{aligned}
&-\frac{\partial}{\partial \xi_{i}}\left(a_{i j}(\xi) \frac{\partial \widehat{u}_{2}(z, \xi, \omega)}{\partial \xi_{j}}\right) \\
&=\left(a_{i j}(\xi)-\widehat{a}_{i j}\right) \frac{\partial^{2} \widehat{u}_{0}(z, \omega)}{\partial z_{i} \partial z_{j}} \\
&+a_{i j}(\xi) \frac{\partial N_{k}(\xi)}{\partial \xi_{j}} \frac{\partial^{2} \widehat{u}_{0}(z, \omega)}{\partial z_{i} \partial z_{k}} \\
&+\frac{\partial}{\partial \xi_{i}}\left(a_{i j}(\xi) N_{k}(\xi)\right) \frac{\partial^{2} \widehat{u}_{0}(z, \omega)}{\partial z_{j} \partial z_{k}} \\
& \quad(z, \xi) \in \Omega \times R^{n}
\end{aligned}
$$

and using the method of separation of variables, we can obtain

$$
\widehat{u}_{2}(z, \xi, \omega)=N_{k l}(\xi) \frac{\partial^{2} \widehat{u}_{0}(z, \omega)}{\partial z_{k} \partial z_{l}},
$$


where $N_{k l}(\xi)(k, l=1, \ldots, n)$ are the solutions of the following problems:

$$
\begin{aligned}
&-\frac{\partial}{\partial \xi_{i}}\left(a_{i j}(\xi) \frac{\partial N_{k l}(\xi)}{\partial \xi_{j}}\right) \\
&= a_{k l}(\xi)-\widehat{a}_{k l}+a_{k j}(\xi) \frac{\partial N_{l}(\xi)}{\partial \xi_{j}} \\
&+\frac{\partial}{\partial \xi_{i}}\left(a_{i k}(\xi) N_{l}(\xi)\right), \quad \xi \in R^{n}, \\
& N_{k l}(\xi) \quad Q \text {-periodic. }
\end{aligned}
$$

Remark 3. Note that $N_{k}(\xi), N_{k l}(\xi)(k, l=1, \ldots, n)$ defined in (11) and (17) can be obtained by resolving the following cell problems in Q:

$$
\begin{array}{r}
-\frac{\partial}{\partial \xi_{i}}\left(a_{i j}(\xi) \frac{\partial N_{k}(\xi)}{\partial \xi_{j}}\right)=\frac{\partial a_{i k}(\xi)}{\partial \xi_{i}}, \quad \xi \in Q \\
N_{k}(\xi) \quad \text { Q-periodic. } \\
-\frac{\partial}{\partial \xi_{i}}\left(a_{i j}(\xi) \frac{\partial N_{k l}(\xi)}{\partial \xi_{j}}\right)=a_{k l}(\xi)-\widehat{a}_{k l}+a_{k j}(\xi) \frac{\partial N_{l}(\xi)}{\partial \xi_{j}} \\
+\frac{\partial}{\partial \xi_{i}}\left(a_{i k}(\xi) N_{l}(\xi)\right), \quad \xi \in Q \\
N_{k l}(\xi) \quad \text { Q-periodic. }
\end{array}
$$

and then extending their solutions by periodicity (see, e.g., [5]), so they are called cell functions as usual.

Therefore, we can obtain the first-order and second-order multiscale asymptotic solution for the space-frequency (4) as

$$
\begin{aligned}
\widehat{U}_{1}^{\varepsilon}(x, \omega)= & \widehat{u}_{0}(z, \omega)+\varepsilon N_{k}(\xi) \frac{\partial \widehat{u}_{0}(z, \omega)}{\partial x_{k}} \\
\widehat{U}_{2}^{\varepsilon}(x, \omega)= & \widehat{u}_{0}(z, \omega)+\varepsilon N_{k}(\xi) \frac{\partial \widehat{u}_{0}(z, \omega)}{\partial x_{k}} \\
& +\varepsilon^{2} N_{k l}(\xi) \frac{\partial^{2} \widehat{u}_{0}(z, \omega)}{\partial x_{k} \partial x_{l}}
\end{aligned}
$$

Remark 4. It is worthwhile to note that all the cell problems are real valued, while the homogenized equation is complex valued. Therefore, when we do numerical computation, we need to repeatedly solve the homogenized problem at a set of specific frequency points but solve all the cell problems only once.

\section{Multiscale Truncation Error Estimate in Frequency Domain}

In order to derive the truncation error estimate for multiscale asymptotic solutions, we first discuss the regularity for the homogenized solution $\widehat{u}_{0}$. The key point is to derive precise estimates for the regularity bounds associated with $\omega$ since the behavior of these bounds as $\omega$ approaches zero and infinity is of critical importance in obtaining the multiscale truncation error estimate and when inverting the multiscale asymptotic solution back to time domain by the inverse Fourier transform.

Proposition 5. Let $\widehat{u}_{0}(\cdot, \omega)$ be the weak solution of the homogenized equation (13) whose coefficients are constants defined in (14). Then,

(i) for any $\widehat{f}(\cdot, \omega), \bar{u} \in L^{2}(\Omega)$, it holds

$$
\begin{gathered}
\left\|\widehat{u}_{0}(\cdot, \omega)\right\|_{0, \Omega} \leq \min \left\{C, \frac{1}{\omega}\right\}\|\widehat{f}(\cdot, \omega)+\bar{u}\|_{0, \Omega}, \\
\left\|\widehat{u}_{0}(\cdot, \omega)\right\|_{1, \Omega} \leq C\|\widehat{f}(\cdot, \omega)+\bar{u}\|_{0, \Omega},
\end{gathered}
$$

where the constant $C$ depending only on $\Omega$ and the coefficients;

(ii) for any $\widehat{f}(\cdot, \omega), \bar{u} \in H^{m}(\Omega)$ and any subdomain $\Omega^{*} \subset \subset$ $\Omega$, it holds

$$
\left\|\widehat{u}_{0}(\cdot, \omega)\right\|_{m+2, \Omega^{*}} \leq C\|\widehat{f}(\cdot, \omega)+\bar{u}\|_{m, \Omega}, \quad m \in \mathscr{Z}^{+},
$$

where the constant $C$ is depending only on $\Omega^{*}, \Omega$ and the coefficients. Moreover, if $\partial \Omega$ is $C^{m+2}$, then we have the global estimate (22) on $\Omega^{*}=\Omega$.

Proof. Define the conjugate bilinear form $\widehat{a}(\cdot, \cdot): H^{1}(\Omega) \times$ $H^{1}(\Omega) \rightarrow \mathscr{C}:$

$$
\widehat{a}(u, v)=\int_{\Omega} \widehat{a}_{i j} \frac{\partial u}{\partial z_{j}} \frac{\partial \widetilde{v}}{\partial z_{i}} d z
$$

where $\widetilde{v}$ denotes the complex conjugate of $v$. Then the variational formulation of the homogenized equation (13) is to find $\widehat{u}_{0} \in H_{0}^{1}(\Omega)$ such that

$$
i \omega\left(\widehat{u}_{0}, v\right)+\widehat{a}\left(\widehat{u}_{0}, v\right)=(\widehat{f}+\bar{u}, v), \quad v \in H_{0}^{1}(\Omega) .
$$

Set $v=\widehat{u}_{0}$ in (24) and take the imaginary part; one obtains

$$
\omega\left\|\widehat{u}_{0}\right\|_{0, \Omega}^{2}=\operatorname{Im}\left\langle\widehat{f}+\bar{u}, \widehat{u}_{0}\right\rangle \leq\|\widehat{f}+\bar{u}\|_{0, \Omega}\left\|\widehat{u}_{0}\right\|_{0, \Omega},
$$

which yields

$$
\left\|\widehat{u}_{0}\right\|_{0, \Omega} \leq \frac{1}{\omega}\|\widehat{f}+\bar{u}\|_{0, \Omega} .
$$

Let $v=\widehat{u}_{0}$ in (24) and consider the real part; with the coercivity of $\widehat{a}_{i j}$ and the Poincare's inequality, we have

$$
\begin{aligned}
\gamma_{1}\left\|\nabla \widehat{u}_{0}\right\|_{0, \Omega}^{2} & \leq \widehat{a}\left(\widehat{u}_{0}, \widehat{u}_{0}\right) \\
& =\operatorname{Re}\left(\widehat{f}+\bar{u}, \widehat{u}_{0}\right) \leq\|\widehat{f}+\bar{u}\|_{0, \Omega}\left\|\widehat{u}_{0}\right\|_{0, \Omega} \\
& \leq C\|\widehat{f}+\bar{u}\|_{0, \Omega}\left\|\nabla \widehat{u}_{0}\right\|_{0, \Omega},
\end{aligned}
$$


and thus

$$
\left\|\widehat{u}_{0}\right\|_{1, \Omega} \leq C\|\widehat{f}+\bar{u}\|_{0, \Omega},
$$

together with (26); we have

$$
\left\|\widehat{u}_{0}\right\|_{0, \Omega} \leq \min \left\{C, \frac{1}{\omega}\right\}\|\widehat{f}+\bar{u}\|_{0, \Omega} .
$$

Next we investigate the interior regularity of the homogenized solution. Fix any open set $\Omega^{*} \subset \subset \Omega$, and choose an open set $W$ such that $\Omega^{*} \subset \subset W \subset \subset \Omega$. Then select a cutoff function $\zeta \in C_{0}^{\infty}\left(R^{n}\right)$ satisfying

$$
\begin{gathered}
\zeta=1, \quad z \in \Omega^{*} ; \\
\zeta=0, \quad z \in R^{n}-W ; \\
0 \leq \zeta \leq 1 .
\end{gathered}
$$

Rewrite (24) as

$$
\widehat{a}\left(\widehat{u}_{0}, v\right)=(\widehat{f}+\bar{u}, v)-i \omega\left(\widehat{u}_{0}, v\right) \equiv B,
$$

and set $v=-D_{k}^{-h}\left(\zeta^{2} D_{k}^{h} \widehat{u}_{0}\right), k \in\{1, \ldots, N\}$, where $D_{k}^{h}$ denotes the difference quotient,

$$
D_{k}^{h} u(z)=\frac{u\left(z+h e_{k}\right)-u(z)}{h}, \quad h \in R, h \neq 0 .
$$

By a standard regularity technique (see, e.g., [18], pages 310313), we have

$$
\widehat{a}\left(\widehat{u}_{0}, v\right) \geq \frac{\gamma_{1}}{2} \int_{\Omega} \zeta^{2}\left|D_{k}^{h} \nabla \widehat{u}_{0}\right|^{2} d z-C \int_{\Omega}\left|\nabla \widehat{u}_{0}\right|^{2} d z .
$$

Cauchy's inequality with $\epsilon$ yields

$$
\begin{aligned}
|B| & \leq C \int_{\Omega}\left(|\widehat{f}+\bar{u}|+\omega\left|\widehat{u}_{0}\right|\right)|v| d z \\
& \leq \epsilon \int_{\Omega}|v|^{2} d z+\frac{C}{\epsilon} \int_{\Omega}\left(|\widehat{f}+\bar{u}|^{2}+\omega^{2}\left|\widehat{u}_{0}\right|^{2}\right) d z,
\end{aligned}
$$

and use the properties of the difference quotient for $v$,

$$
\int_{\Omega}|v|^{2} d z \leq C \int_{\Omega}\left(\left|\nabla \widehat{u}_{0}\right|^{2}+\zeta^{2}\left|D_{k}^{h} \nabla \widehat{u}_{0}\right|^{2}\right) d z,
$$

and choose $\epsilon=\gamma_{1} / 4$ that yields the bound

$$
\begin{aligned}
|B| \leq & \frac{\gamma_{1}}{4} \int_{\Omega} \zeta^{2}\left|D_{k}^{h} \nabla \widehat{u}_{0}\right|^{2} d z \\
& +C \int_{\Omega}|\widehat{f}+\bar{u}|^{2}+\omega^{2}\left|\widehat{u}_{0}\right|^{2}+\left|\nabla \widehat{u}_{0}\right|^{2} d z .
\end{aligned}
$$

Combining (33) and (36) implied

$$
\begin{aligned}
\int_{\Omega^{*}}\left|D_{k}^{h} \nabla \widehat{u}_{0}\right|^{2} d z & \leq C \int_{\Omega} \zeta^{2}\left|D_{k}^{h} \nabla \widehat{u}_{0}\right|^{2} d z \\
& \leq C \int_{\Omega}\left(|\widehat{f}+\bar{u}|^{2}+\omega^{2}\left|\widehat{u}_{0}\right|^{2}+\left|\nabla \widehat{u}_{0}\right|^{2}\right) d z .
\end{aligned}
$$

In view of the properties of the difference quotient, with (29) and (28), we deduce $\widehat{u}_{0} \in H_{\text {loc }}^{2}$, with the estimate

$$
\begin{aligned}
\left\|\widehat{u}_{0}\right\|_{2, \Omega^{*}} & \leq C\left(\|\hat{f}+\bar{u}\|_{0, \Omega}+\omega\left\|\widehat{u}_{0}\right\|_{0, \Omega}+\left\|\nabla \widehat{u}_{0}\right\|_{0, \Omega}\right) \\
& \leq C\|\hat{f}+\bar{u}\|_{0, \Omega} .
\end{aligned}
$$

For higher regularity of $\widehat{u}_{0}$, insert $v=-\partial w / \partial z_{k}$ into (24), $k=1, \ldots, n$, where $w \in C^{\infty}(\Omega)$, and perform integration by parts; we have

$$
i \omega\left(\frac{\partial \widehat{u}_{0}}{\partial z_{k}}, w\right)+\widehat{a}\left(\frac{\partial \widehat{u}_{0}}{\partial z_{k}}, w\right)=\left(\frac{\partial(\widehat{f}+\bar{u})}{\partial z_{k}}, w\right) .
$$

In view of (38), applying the interior $H^{2}$ regularity result to $\partial \widehat{u}_{0} / \partial z_{k}$, then

$$
\left\|\frac{\partial \widehat{u}_{0}}{\partial z_{k}}\right\|_{2, \Omega^{*}} \leq C\left\|\frac{\partial(\hat{f}+\bar{u})}{\partial z_{k}}\right\|_{0, \Omega}, \quad k=1, \ldots, n,
$$

which yields

$$
\left\|\widehat{u}_{0}\right\|_{3, \Omega^{*}} \leq C\|\widehat{f}+\bar{u}\|_{1, \Omega} .
$$

Finally we can obtain (22) by induction on $m$. Given $\partial \Omega \epsilon$ $\mathrm{C}^{m+2}$, following the standard boundary regularity technique we get the global estimate

$$
\left\|\widehat{u}_{0}(\cdot, \omega)\right\|_{m+2, \Omega} \leq C\|\hat{f}(\cdot, \omega)+\bar{u}\|_{m, \Omega} .
$$

Next we can derive the truncation error estimate for multiscale asymptotic solutions (20) in frequency domain.

Theorem 6. Let $\widehat{u}^{\varepsilon}(x, \omega)$ be the weak solution of (4), and let $\widehat{U}_{s}^{\varepsilon}(x, \omega)(s=1,2)$ be the multiscale asymptotic solutions given in (20). Under the assumptions $\left(A_{1}\right)-\left(A_{3}\right)$, with $\widehat{f}(\cdot, \omega) \in$ $H^{1}(\Omega), \bar{u} \in H^{1}(\Omega), \widehat{u}_{0}(\cdot, \omega) \in H^{3}(\Omega)$, there exists a constant $C$ independent of $\varepsilon$ and $\omega$ such that

$$
\begin{aligned}
& \left\|\widehat{u}^{\varepsilon}(\cdot, \omega)-\widehat{U}_{2}^{\varepsilon}(\cdot, \omega)\right\|_{1, \Omega} \\
& \quad \leq C(1+\omega) \varepsilon^{1 / 2}\|\widehat{f}(\cdot, \omega)+\bar{u}\|_{1, \Omega}, \quad s=1,2 .
\end{aligned}
$$

Proof. For $s=2$, set $Z_{\varepsilon}(x, \omega)=\widehat{u}^{\varepsilon}(x, \omega)-\widehat{U}_{2}^{\varepsilon}(x, \omega)$; one can check $Z_{\varepsilon}(x, \omega)$ that satisfy

$$
\begin{gathered}
i \omega Z_{\varepsilon}(x, \omega)-\frac{\partial}{\partial x_{i}}\left(a_{i j}\left(\frac{x}{\varepsilon}\right) \frac{\partial Z_{\varepsilon}(x, \omega)}{\partial x_{j}}\right) \\
=\varepsilon F^{\varepsilon}(x, \omega), \quad x \in \Omega, \\
Z_{\varepsilon}(x, \omega)=\varepsilon G^{\varepsilon}(x, \omega), \quad x \in \partial \Omega,
\end{gathered}
$$


where

$$
\begin{gathered}
F^{\varepsilon} \equiv F_{0}^{\varepsilon}+\varepsilon \frac{\partial F_{i}^{\varepsilon}}{\partial x_{i}} \\
F_{0}^{\varepsilon}=\left(a_{i l}(\xi) N_{k}(\xi)+a_{i j}(\xi) \frac{\partial N_{k l}(\xi)}{\partial \xi_{j}}\right) \frac{\partial^{3} \widehat{u}_{0}(z, \omega)}{\partial z_{i} \partial z_{k} \partial z_{l}} \\
-i \omega N_{k}(\xi) \frac{\partial \widehat{u}_{0}(z, \omega)}{\partial z_{k}}-i \omega \varepsilon N_{k l}(\xi) \frac{\partial^{2} \widehat{u}_{0}(z, \omega)}{\partial z_{k} \partial z_{l}} \\
F_{i}^{\varepsilon}=a_{i j}(\xi) N_{k l}(\xi) \frac{\partial^{3} \widehat{u}_{0}(z, \omega)}{\partial z_{j} \partial z_{k} \partial z_{l}} \\
G^{\varepsilon}=-N_{k}(\xi) \frac{\partial \widehat{u}_{0}(z, \omega)}{\partial z_{k}}-\varepsilon N_{k l}(\xi) \frac{\partial^{2} \widehat{u}_{0}(z, \omega)}{\partial z_{k} \partial z_{l}} .
\end{gathered}
$$

Under the assumptions $\left(A_{1}\right)-\left(A_{3}\right)$, it follows from Theorem 1.1 of [2] (also see [1]) that

$$
\left\|N_{k}\right\|_{1, \infty, Q} \leq C, \quad\left\|N_{k l}\right\|_{1, \infty, Q} \leq C, \quad k, l=1, \ldots, n .
$$

Together with the regularity estimate of $\widehat{u}_{0}$, one can obtain

$$
\begin{gathered}
\left\|F_{0}^{\varepsilon}\right\|_{0, \Omega} \leq C(1+\omega)\|\hat{f}+\bar{u}\|_{1, \Omega}, \\
\left\|F_{i}^{\varepsilon}\right\|_{0, \Omega} \leq C\|\hat{f}+\bar{u}\|_{1, \Omega},
\end{gathered}
$$

and therefore, we get $F^{\varepsilon} \in H^{-1}(\Omega)$ and

$$
\left\|F^{\varepsilon}\right\|_{-1, \Omega} \leq C(1+\omega)\|\widehat{f}+\bar{u}\|_{1, \Omega} .
$$

In order to get the boundary estimate, introducing the following cutoff function $m_{\varepsilon} \in C_{0}^{\infty}(\Omega)$ such that

$$
\begin{gathered}
m_{\varepsilon}=1, \quad \text { if } \operatorname{dist}(x, \partial \Omega) \leq \varepsilon, \\
m_{\varepsilon}=0, \quad \text { if } \operatorname{dist}(x, \partial \Omega) \geq 2 \varepsilon, \\
\left\|\nabla m_{\varepsilon}\right\|_{L^{\infty}(\Omega)} \leq C \varepsilon^{-1} ;
\end{gathered}
$$

and setting $\Psi^{\varepsilon}=m_{\varepsilon} G^{\varepsilon}, \Omega_{\varepsilon}=\operatorname{supp} \Psi^{\varepsilon}$, with the regularity estimate of $\widehat{u}_{0}$, we can obtain

$$
\begin{gathered}
\left\|\Psi^{\varepsilon}\right\|_{0, \Omega_{\varepsilon}} \leq C\|\hat{f}+\bar{u}\|_{0, \Omega} \\
\left\|\frac{\partial \Psi^{\varepsilon}}{\partial x_{i}}\right\|_{0, \Omega_{\varepsilon}} \leq C \varepsilon^{-1}\left\|\widehat{u}_{0}\right\|_{1, \Omega_{\varepsilon}}+C\left\|\widehat{u}_{0}\right\|_{3, \Omega_{\varepsilon}} .
\end{gathered}
$$

Using the following estimate on boundary layer $\Omega_{\varepsilon}$, (see [6])

$$
\left\|\widehat{u}_{0}\right\|_{1, \Omega_{\varepsilon}} \leq C \varepsilon^{1 / 2}\left\|\nabla \widehat{u}_{0}\right\|_{1, \Omega}
$$

we have

$$
\left\|\Psi^{\varepsilon}\right\|_{1, \Omega_{\varepsilon}} \leq C \varepsilon^{-1 / 2}\|\widehat{f}+\bar{u}\|_{1, \Omega} .
$$

By the trace theorem, we know

$$
\begin{aligned}
\left\|G^{\varepsilon}\right\|_{1 / 2, \partial \Omega} & =\left\|\Psi^{\varepsilon}\right\|_{1 / 2, \partial \Omega} \leq C\left\|\Psi^{\varepsilon}\right\|_{1, \Omega_{\varepsilon}} \\
& \leq C \varepsilon^{-1 / 2}\|\widehat{f}+\bar{u}\|_{1, \Omega} .
\end{aligned}
$$

Similar to Proposition 5 for (44) with nonhomogeneous boundary condition, we can drive the following estimate:

$$
\left\|Z_{\varepsilon}\right\|_{1, \Omega} \leq C \varepsilon\left\|F^{\varepsilon}\right\|_{-1, \Omega}+C(1+\omega) \varepsilon\left\|G^{\varepsilon}\right\|_{1 / 2, \partial \Omega},
$$

together with (48) and (53) and we have

$$
\left\|\widehat{u}^{\varepsilon}(\cdot, \omega)-\widehat{U}_{2}^{\varepsilon}(\cdot, \omega)\right\|_{1, \Omega} \leq C(1+\omega) \varepsilon^{1 / 2}\|\widehat{f}+\bar{u}\|_{1, \Omega},
$$

where $C$ is independent of $\varepsilon$ and $\omega$.

For $s=1$, by means of Theorem 1.2 in [6], we can also derive the estimate (43). Therefore the proof is complete.

Remark 7. It is worth noting that, for any $\Omega^{*} \subset \subset \Omega$, it is well known that homogenized solution $\widehat{u}_{0} \in H^{3}\left(\Omega^{*}\right)$ if $\widehat{f}(\cdot, \omega)$, and $\bar{u} \in H^{1}(\Omega)$. If $\widehat{u}_{0}$ does not have the local $H^{3}$ regularity near the boundary $\partial \Omega$, follow the way in [19]; we can use the asymptotic expansion in the interior subdomain and construct the boundary layer near $\partial \Omega$.

\section{Inverse Fourier Transform}

In this section, we will construct the approximate solution of the parabolic equation (1) in space-time domain by the inverse Fourier transform. Recall the formula of the inverse Fourier transform as

$$
\begin{aligned}
v(\cdot, t) & =\frac{1}{2 \pi} \int_{-\infty}^{+\infty} \widehat{v}(\cdot, \omega) e^{i \omega t} d \omega \\
& =\frac{1}{\pi} \operatorname{Re} \int_{0}^{\infty} \widehat{v}(\cdot, \omega) e^{i \omega t} d \omega,
\end{aligned}
$$

provided that $v(\cdot, t)$ is a real function.

Fix a sufficiently large $\omega^{*}>0$ such that $u^{\varepsilon}(\cdot, \omega)$ is negligible for $|\omega|>\omega^{*}$. Set $\Delta \omega=\omega^{*} / M$, where $M \in \mathscr{Z}^{+}$ is a selected segment number for the interval $\left(0, \omega^{*}\right)$ and the space-time solution $u^{\varepsilon}$ of (1) can be approximated by

$$
U_{s, \omega^{*}, \Delta \omega}^{\varepsilon}(x, t)=\frac{1}{\pi} \operatorname{Re} \sum_{j=1}^{M} \widehat{U}_{s}^{\varepsilon}\left(x, \omega_{j-1 / 2}\right) e^{i t \omega_{j-1 / 2}} \Delta \omega,
$$

where $\omega_{j-1 / 2}=(j-1 / 2) \Delta \omega, j=1, \ldots, M$.

In order to get an estimate for $u^{\varepsilon}-U_{s, \omega^{*}, \Delta \omega}^{\varepsilon}$, let

$$
\begin{gathered}
u_{\omega^{*}}^{\varepsilon}(x, t)=\frac{1}{\pi} \operatorname{Re} \int_{0}^{\omega^{*}} \widehat{u}^{\varepsilon}(x, \omega) e^{i \omega t} d \omega, \\
u_{\omega^{*}, \Delta \omega}^{\varepsilon}(x, t)=\frac{1}{\pi} \operatorname{Re} \sum_{j=1}^{M} \widehat{u}^{\varepsilon}\left(x, \omega_{j-1 / 2}\right) e^{i t \omega_{j-1 / 2}} \Delta \omega,
\end{gathered}
$$

and we get

$$
\begin{aligned}
u^{\varepsilon}(x, t) & -U_{s, \omega^{*}, \Delta \omega}^{\varepsilon}(x, t) \\
= & \left(u^{\varepsilon}(x, t)-u_{\omega^{*}}^{\varepsilon}(x, t)\right) \\
& +\left(u_{\omega^{*}}^{\varepsilon}(x, t)-u_{\omega^{*}, \Delta \omega}^{\varepsilon}(x, t)\right)+ \\
& +\left(u_{\omega^{*}, \Delta \omega}^{\varepsilon}(x, t)-U_{s, \omega^{*}, \Delta \omega}^{\varepsilon}(x, t)\right) \\
\equiv & E_{1}(x, t)+E_{2}(x, t)+E_{3}(x, t) .
\end{aligned}
$$


For $E_{1}$, from Proposition 5, we can check

$$
\begin{aligned}
\left\|E_{1}(\cdot, t)\right\|_{0, \Omega} & \leq \frac{1}{\pi} \int_{\omega^{*}}^{\infty}\left\|\widehat{u}^{\varepsilon}(\cdot, \omega)\right\|_{0, \Omega} d \omega \\
& \leq \frac{1}{\pi} \int_{\omega^{*}}^{\infty} \frac{1}{\omega}\|\widehat{f}(\cdot, \omega)+\bar{u}\|_{0, \Omega} d \omega .
\end{aligned}
$$

For the estimate of $E_{2}$, the following lemma was proved in [17].

Lemma 8. Let

$$
\begin{gathered}
F_{k}^{0}(t)=\left(\int_{0}^{t} e^{t-\sigma}\left\|\sigma^{k} f(x, \sigma)\right\|^{2} d \sigma\right)^{1 / 2}, \\
F_{k}^{l}(t) \\
=\left(\int_{0}^{t} \int_{0}^{\sigma_{l}} \cdots \int_{0}^{\sigma_{1}} e^{t-\sigma}\left\|\sigma^{k-l} f(x, \sigma)\right\|^{2} d \sigma d \sigma_{1} \cdots d \sigma_{l}\right)^{1 / 2},
\end{gathered}
$$

and suppose that for $k=0,1,2, F_{k}^{l}(t) \in L^{2}(J)$ for all $k \geq l \geq 0$. Then

$$
\left\|E_{2}(\cdot, t)\right\|_{0, \Omega} \leq C \Delta \omega^{2} \sum_{k=0}^{2} t^{2-k} \sum_{l=0}^{k}\left\|F_{k}^{l}(t)\right\|_{0, \Omega} .
$$

For $E_{3}$, by Theorem 6, we have

$$
\begin{aligned}
& \left\|E_{3}(\cdot, t)\right\|_{0, \Omega} \\
& =\left\|\frac{1}{\pi} \operatorname{Re} \sum_{j=1}^{M}\left(\widehat{u}^{\varepsilon}\left(x, \omega_{j-1 / 2}\right)-\widehat{U}_{s}^{\varepsilon}\left(x, \omega_{j-1 / 2}\right)\right) e^{i t \omega_{j-1 / 2}} \Delta \omega\right\|_{0, \Omega} \\
& \leq C \Delta \omega \sum_{j=1}^{M}\left(1+\omega_{j-1 / 2}\right) \varepsilon^{1 / 2}\left\|\widehat{f}\left(\cdot, \omega_{j-1 / 2}\right)+\bar{u}\right\|_{1, \Omega} \\
& \leq C \varepsilon^{1 / 2} \int_{0}^{\omega^{*}}(1+\omega)\|\hat{f}(\cdot, \omega)+\bar{u}\|_{1, \Omega} d \omega .
\end{aligned}
$$

Combining (60), (62), and (63), we have the following theorem for the full error estimate.

Theorem 9. Let $u^{\varepsilon}(\cdot, t)$ be the solution of (1), and let $U_{s, \omega^{*}, \Delta \omega}^{\varepsilon}(\cdot, t)(s=1,2)$ be the approximate solution given in (57). Under the assumptions $\left(A_{1}\right)-\left(A_{3}\right), \widehat{f}(\cdot, \omega), \bar{u} \in H^{1}(\Omega)$, $\widehat{u}_{0}(\cdot, \omega) \in H^{3}(\Omega)$, and suppose that for $k=0,1,2, F_{k}^{l}(t)$ defined in (61) belong to $L^{2}(J)$ for all $k \geq l \geq 0$; there exists a constant $C$ independent of $\varepsilon$ and $\omega$ such that

$$
\begin{aligned}
\| u^{\varepsilon}(\cdot, t) & -U_{s, \omega^{*}, \Delta \omega}^{\varepsilon}(\cdot, t) \|_{0, \Omega} \\
\leq & \frac{1}{\pi} \int_{\omega^{*}}^{\infty} \frac{1}{\omega}\|\widehat{f}(\cdot, \omega)+\bar{u}\|_{0, \Omega} d \omega \\
& +C \Delta \omega^{2} \sum_{k=0}^{2} t^{2-k} \sum_{l=0}^{k}\left\|F_{k}^{l}(t)\right\|_{0, \Omega} \\
& +C \varepsilon^{1 / 2} \int_{0}^{\omega^{*}}(1+\omega)\|\widehat{f}(\cdot, \omega)+\bar{u}\|_{1, \Omega} d \omega .
\end{aligned}
$$

\section{Parallel Multiscale FEM Algorithm and Numerical Tests}

In simulation, we will apply the following first-order and second-order difference quotients of some functions $f(x)$ given by

$$
\delta_{x_{j}} f^{h}\left(N_{p}\right)=\frac{1}{\tau\left(N_{p}\right)} \sum_{e \in \sigma\left(N_{p}\right)}\left[\frac{\partial f^{h}}{\partial x_{j}}\right]_{e}\left(N_{p}\right),
$$

$$
\begin{array}{r}
\delta_{x_{l} x_{m}}^{2} f^{h}\left(N_{p}\right) \\
=\frac{1}{\tau\left(N_{p}\right)} \sum_{e \in \sigma\left(N_{p}\right)}\left[\sum_{j=1}^{d} \delta_{x_{l}} f^{h}\left(P_{j}\right) \frac{\partial x_{j}}{\partial x_{m}}\right]_{e}\left(N_{p}\right), \\
j, l, m=1, \ldots, n,
\end{array}
$$

where $\sigma\left(N_{p}\right)$, the set of elements with the node $N_{p} ; \tau\left(N_{p}\right)$, is the number of elements of $\sigma\left(N_{p}\right) ; d$ is the number of nodes on the element $e ; P_{j}$ are the nodes of the element $e ; \chi_{j}(x)$ are the Lagrangian shape functions; $f^{h}$ denotes the FEM solution of $f ;\left[\partial f / \partial x_{j}\right]_{e}\left(N_{p}\right)$ is the value of $\partial f / \partial x_{j}$ at the node $N_{p}$ associated with the element $e$.

The following parallel multiscale FEM algorithm are presented to solve (1).

Step 1. Solve the first-order cell problems of $N_{k}$ defined in (18) for $k=1, \ldots, n$ and the second-order cell problems of $N_{k l}$ defined in (19) for $k, l=1, \ldots, n$ in local fine grid of the periodic cell $Q$ by FEM.

Step 2. Compute the homogenized coefficients $\widehat{a}_{i j}$ defined in (14) in the local fine grid of the periodic cell $Q$ by numerical integration.

Step 3. Fix a sufficiently large $\omega^{*}>0$ and set $\Delta \omega=\omega^{*} / M$, $M \in \mathscr{Z}^{+}$and simultaneously solve the complex-valued homogenized equation (13) in the whole domain $\Omega$ in a coarse mesh at $\omega_{j-1 / 2}=(j-1 / 2) \Delta \omega, j=1, \ldots, M$. Then compute the first-order and second-order partial derivatives of the homogenized solutions by high order difference quotients in the same coarse mesh.

Step 4. Extend all the cell functions by periodicity from the local fine grid of the periodic cell $Q$ to the gird of the whole domain $\Omega$. Then compute the multiscale asymptotic solutions given by (20) at frequency points $\omega_{j-1 / 2}, j=1, \ldots, M$.

Step 5. Calculate the approximation solutions for any fixed time $t=t^{*}$ for the original equation (1) according to the formula (57).

Remark 10. Notice that cell problems are independent of the time variable $t$ or the frequency variable $\omega$, so they only need to be solved once.

Remark 11. It should be stated that Step 1 to Step 5 in the algorithm are serial. However, for the time-dependent problems, the main computation of the existing multiscale algorithms 


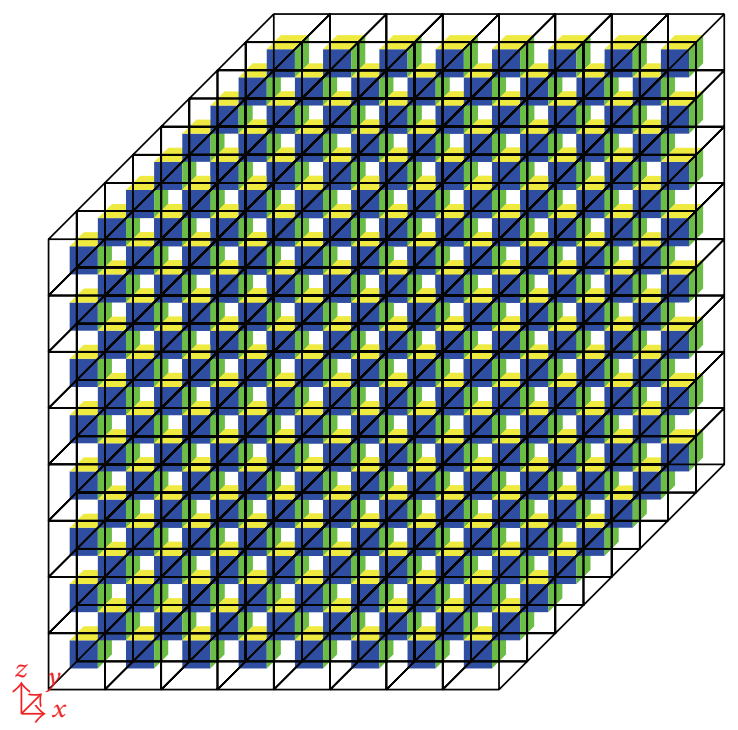

(a)

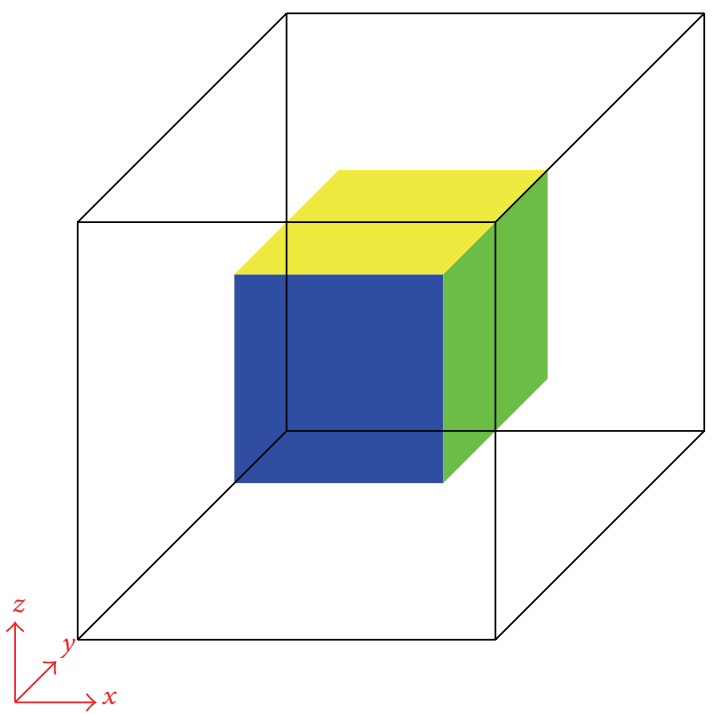

(b)

Figure 1: (a) A whole domain $\Omega$, (b) the reference cell $Q$.

is in computing the time-dependent homogenized equation step by step. In our presented algorithm, we can simultaneously solve the homogenized equations at different frequent points, so the main computation of the algorithm is parallel. Also, notice that each step can be done simultaneously. In this respect, different from other multiscale algorithms for the time-dependent equation, we called it parallel algorithm.

Remark 12. If some conditions of the geometric symmetry are satisfied (see [19]), then we can employ the homogeneous Dirichlet boundary conditions on the boundary $\partial Q$ for the cell problems (18) and (19). Following the way in [19], one can also obtain a similar convergence result to that of Theorem 6 .

Now we discuss the finite element computation for the complex-valued homogenized Equation (13) in $\Omega$. Let $\widehat{u}_{0, i}=$ $\widehat{u}_{0, R}+\widehat{u}_{0, I}$ and $\widehat{f}=\widehat{f}_{R}+\widehat{f}_{I}$, where the subscript $R$ denotes the real part of a function and the subscript $I$ denotes the imaginary part.

The variational form of $(13)$ is to find that $\left(\widehat{u}_{0, R}, \widehat{u}_{0, I}\right) \in$ $H_{0}^{1}(\Omega) \times H_{0}^{1}(\Omega)$ satisfied

$$
\begin{gathered}
-\omega\left(\widehat{u}_{0, I}, v\right)+\widehat{a}\left(\widehat{u}_{0, R}, v\right)=\left(\widehat{f}_{R}+\bar{u}, v\right), \\
\omega\left(\widehat{u}_{0, R}, v\right)+\widehat{a}\left(\widehat{u}_{0, I}, v\right)=\left(\widehat{f}_{I}, v\right)
\end{gathered}
$$

for any $v \in H_{0}^{1}(\Omega)$, and $\widehat{a}(\cdot, \cdot)$ is given by $(23)$. Let $S_{h} \subset H_{0}^{1}(\Omega)$ be the finite element space, the discrete variational form of (66) is to find that $\left(\widehat{u}_{0, R}^{h}, \widehat{u}_{0, I}^{h}\right) \in S_{h} \times S_{h}$ satisfied

$$
\begin{gathered}
-\omega\left(\widehat{u}_{0, I}^{h}, v^{h}\right)+\widehat{a}\left(\widehat{u}_{0, R}^{h}, v^{h}\right)=\left(\widehat{f}_{R}+\bar{u}, v^{h}\right), \\
\omega\left(\widehat{u}_{0, R}^{h}, v^{h}\right)+\widehat{a}\left(\widehat{u}_{0, I}^{h}, v^{h}\right)=\left(\widehat{f}_{I}, v^{h}\right),
\end{gathered}
$$

for any $v^{h} \in S_{h}$. Let $\left\{\phi_{j}(x)\right\}_{j=1}^{N_{h}}$ be a set of basis of $S_{h}$, and set

$$
\widehat{u}_{0, R}^{h}(x, P)=\sum_{j=1}^{N_{h}} \Gamma_{R_{j}} \phi_{j}(x), \quad \widehat{u}_{0, I}^{h}(x, P)=\sum_{j=1}^{N_{h}} \Gamma_{I_{j}} \phi_{j}(x),
$$

and therefore, we solve the linear system in dimension $2 * N_{h}$ as follows:

$$
\left(\begin{array}{cc}
A & -\omega B \\
\omega B & A
\end{array}\right)\left(\begin{array}{c}
\boldsymbol{\Gamma}_{R} \\
\boldsymbol{\Gamma}_{I}
\end{array}\right)=\left(\begin{array}{c}
\mathbf{F}_{R} \\
\mathbf{F}_{I}
\end{array}\right)
$$

where

$$
\begin{gathered}
A=\left\{\widehat{a}\left(\phi_{j}, \phi_{k}\right)\right\}_{1 \leq j, k \leq N_{h}}, \\
B=\left\{\left(\phi_{j}, \phi_{k}\right)\right\}_{1 \leq j, k \leq N_{h}}, \\
\Gamma_{R}:=\left(\Gamma_{R_{1}}, \ldots, \Gamma_{R_{N_{h}}}\right)^{T}, \quad \Gamma_{I}:=\left(\Gamma_{I_{1}}, \ldots, \Gamma_{I_{N_{h}}}\right)^{T}, \\
\mathbf{F}_{R}:=\left(\left(\widehat{f}_{R}+\bar{u}, \phi_{1}\right), \ldots,\left(\widehat{f}_{R}+\bar{u}, \phi_{N_{h}}\right)\right)^{T}, \\
\mathbf{F}_{I}:=\left(\left(\widehat{f}_{I}, \phi_{1}\right), \ldots,\left(\widehat{f}_{I}, \phi_{N_{h}}\right)\right)^{T} .
\end{gathered}
$$

To validate the proposed method and to confirm the theoretical analysis reported in this paper, we present numerical simulations for the following cases.

Example. Consider the parabolic equation (1) in 3D composite materials, where whole domain $\Omega$ and the periodic cell $Q$ are illustrated in Figures 1(a) and 1(b). In the configuration, we take $\varepsilon=1 / 8$ and the blue cube $[0.25,0.75]^{3}$ denotes 


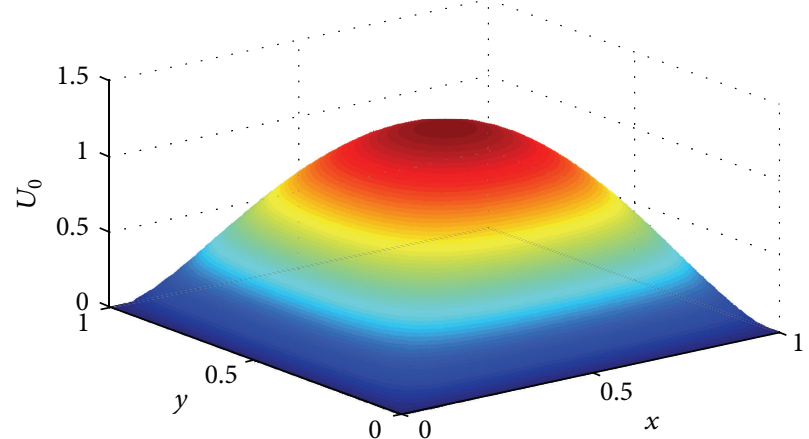

(a)

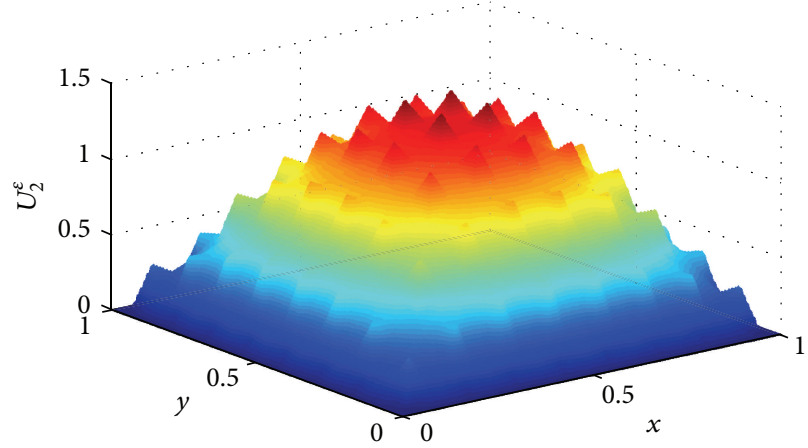

(c)

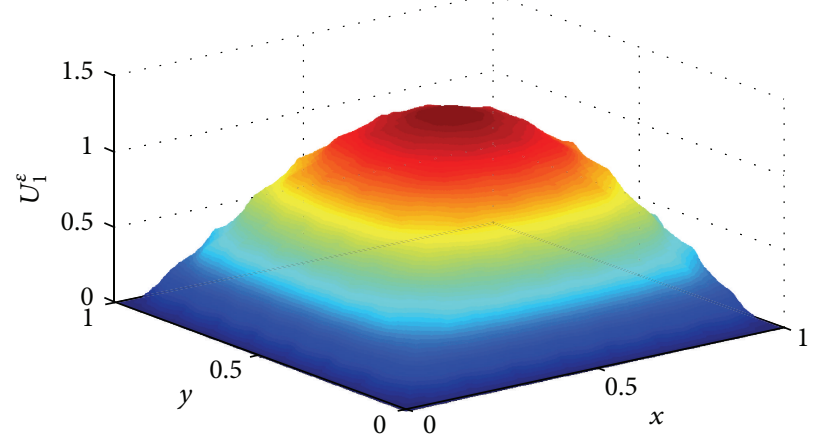

(b)

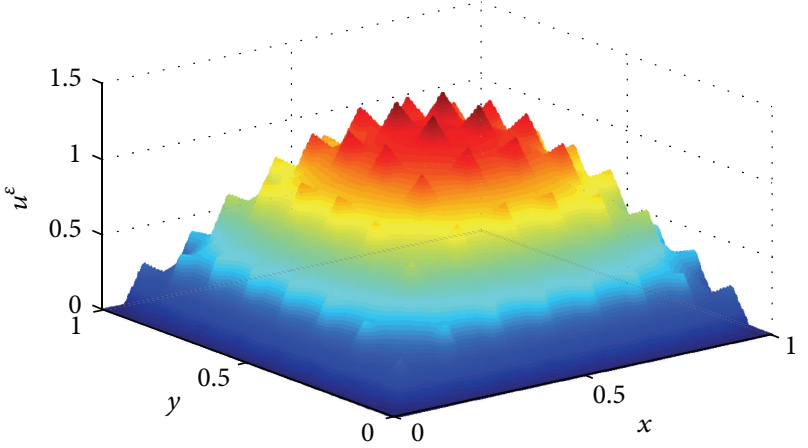

(d)

Figure 2: Case 1: The solutions in the cross-section $z=0.5625, t=0.1, \omega^{*}=100$, and $M=100$. (a) The homogenized solution $U_{0}$; (b) the first-order multiscale solution $U_{1}^{\varepsilon}$; (c) the second-order multiscale solution $U_{2}^{\varepsilon}$; (d) the reference solution $u^{\varepsilon}$.

the inclusion and other parts are the matrix. The parameters of the composite materials for two cases will be considered,

$$
\begin{aligned}
& \text { Case 1: } a_{i j}(\xi)= \begin{cases}10 \delta_{i j} & \text { in inclusion } \\
500 \delta_{i j} & \text { in matrix, }\end{cases} \\
& \text { Case 2: } a_{i j}(\xi)= \begin{cases}500 \delta_{i j} & \text { in inclusion } \\
10 \delta_{i j} & \text { in matrix, }\end{cases}
\end{aligned}
$$

where $\xi=\varepsilon^{-1} x$. Let $f(x)=e^{-t}$ and $\bar{u}(x)=0$.

To assess the validity of the presented method, the exact solution $u^{\varepsilon}$ of the original parabolic equation (1) must be available. It is extremely difficult and even impossible to find the exact solution; we replace it by the numerical solution fully resolved by FEM in space and FDM in time using fine meshes (FD-FE method). It should be noted that more considerable computing time will be required to solve (1) using fully resolved FD-FE method when $\varepsilon$ is small, so we only present numerical results for $\varepsilon=1 / 8$. Here, the linear tetrahedral elements are employed for the semidiscrete problem of (1) using fine meshes, and the solution is computed by the Euler midpoint scheme.

Without confusion, let $u^{\varepsilon}$ denote the numerical solution for the original problem (1) using the fully resolved FDFE method. Let $U_{1}^{\varepsilon}, U_{2}^{\varepsilon}$ denote the numerical solutions inverted back by the first-order and second-order multiscale asymptotic solutions in accordance with (57), respectively. Also, let $U_{0}$ denote the numerical solution inverted back by the homogenized solutions according to the following formula:

$$
U_{0, \omega^{*}, \Delta \omega}(x, t)=\frac{1}{\pi} \operatorname{Re} \sum_{j=1}^{M} \widehat{u}_{0}\left(x, \omega_{j-1 / 2}\right) e^{i t \omega_{j-1 / 2}} \Delta \omega .
$$

All the codes are implemented on parallel hierarchical grid (PHG), which is a toolbox for developing parallel adaptive finite element programs. PHG is currently under active development at State Key Laboratory of Scientific and Engineering Computing of CAS. We use linear tetrahedron elements to solve the related problems numerically.

The numerical results in the cross-section $z=0.5625$ and at time $t=0.1$ for Case 1 and Case 2 are displayed in Figures 2 and 3, respectively. Although the first-order and second-order multiscale asymptotic solutions have the same convergence rate as stated in Theorem 9, numerical results displayed in Figure 2 confirm that the second-order corrector terms are crucial in some cases.

The relative errors vary with the segment number $M$ in the numerical integration formula (57) for homogenized method (HM), first-order multiscale method (1st MsM), and second-order multiscale method (2nd MsM) corresponding to $U_{0}, U_{1}^{\varepsilon}$, and $U_{2}^{\varepsilon}$ that are listed in Figure 4. We observe that the relative errors become stable after $M=80$. Also, the relative errors evolution over time is listed in Figure 5. The results show that the accuracy of the proposed method especially the 2 nd MsM is in good agreement with the fully 


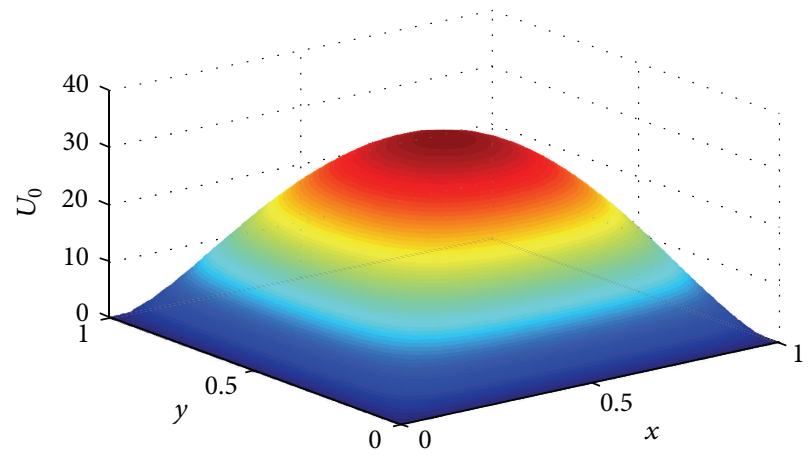

(a)

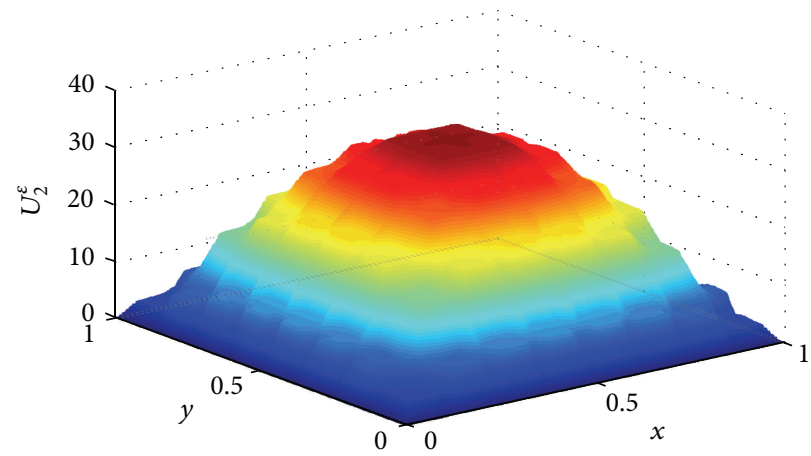

(c)

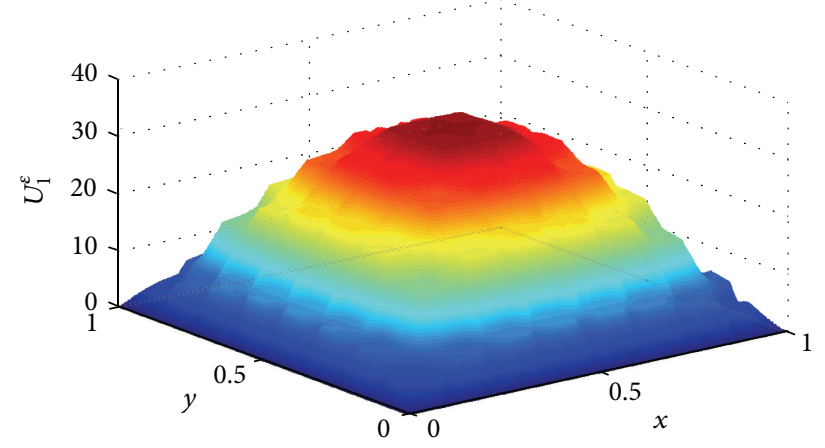

(b)

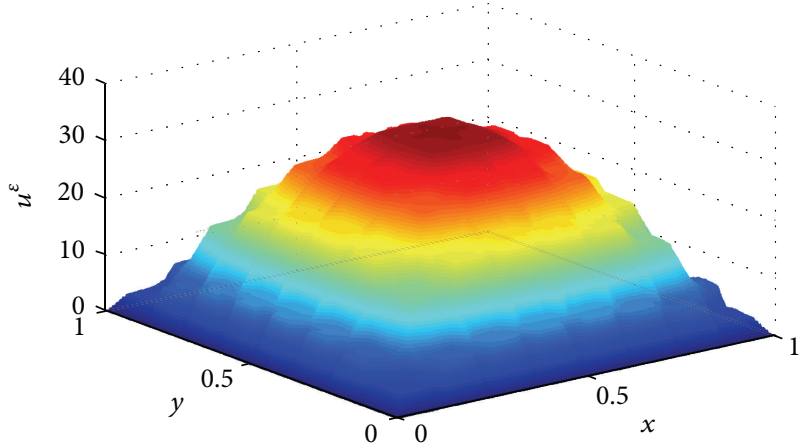

(d)

FIgUre 3: Case 2: The solutions in the cross-section $z=0.5625, t=0.1, \omega^{*}=100$, and $M=100$. (a) The homogenized solution $U_{0}$; (b) the first-order multiscale solution $U_{1}^{\varepsilon}$; (c) the second-order multiscale solution $U_{2}^{\varepsilon}$; (d) the reference solution $u^{\varepsilon}$.

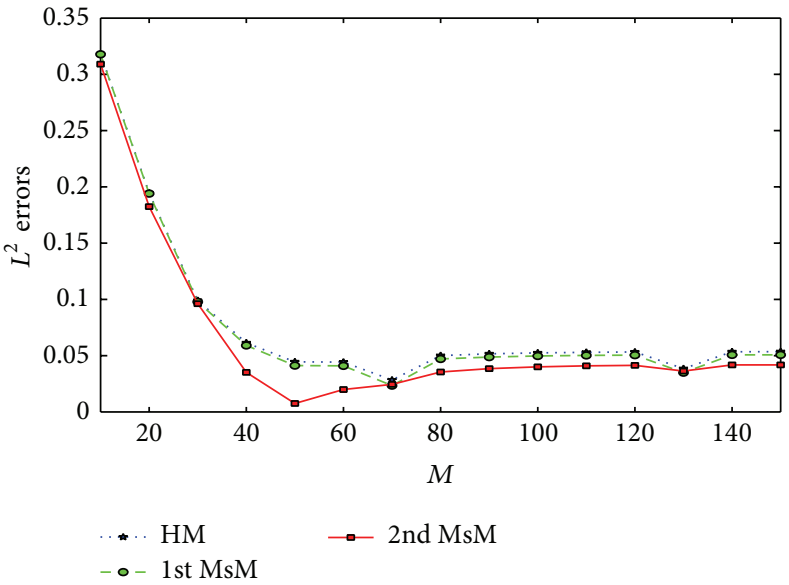

(a)

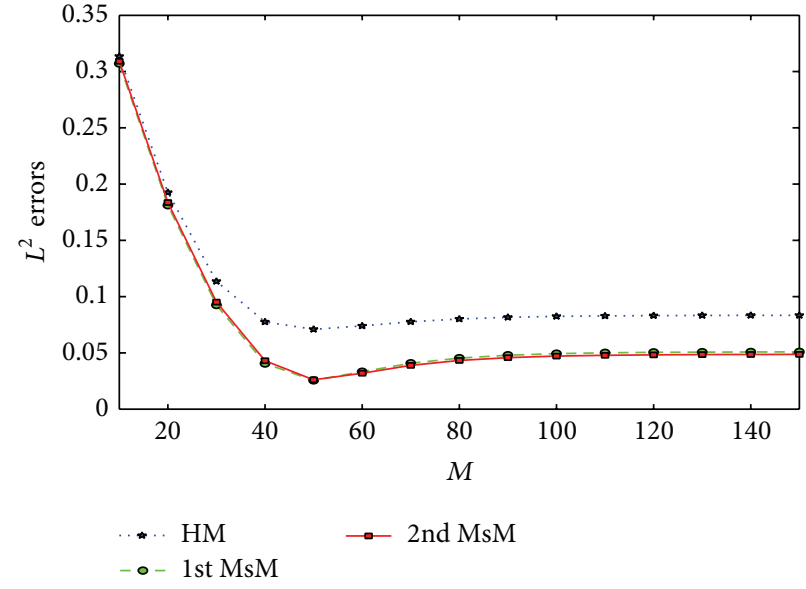

(b)

FIGURE 4: $L^{2}$-norm relative errors vary with the segment number $M$ at $t=0.1, \omega^{*}=100$ (a) Case 1; (b) Case 2.

resolved case. It is worthwhile to note that the relative errors do not grow rapidly as time increases.

The costs required by the developed multiscale finite element method are not sensitive to the time variable but lie on the number of space-frequency equations which is determined by the segment number $M$, and each spacefrequency equation is solved by multiscale finite element method with the computational cost listed in Table 1; for the fully resolved case, we need to compute $T / \Delta t$ steps and the cost for each time step is also listed in Table 1. 


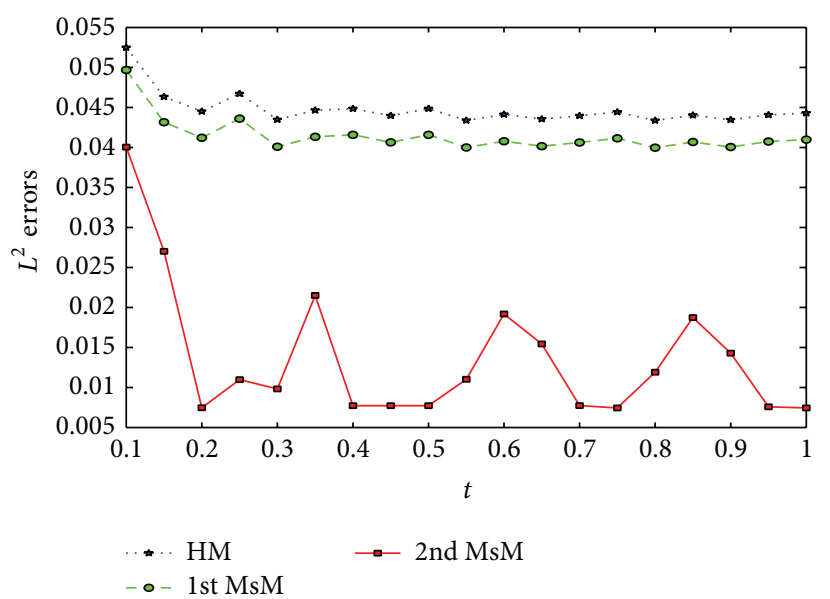

(a)

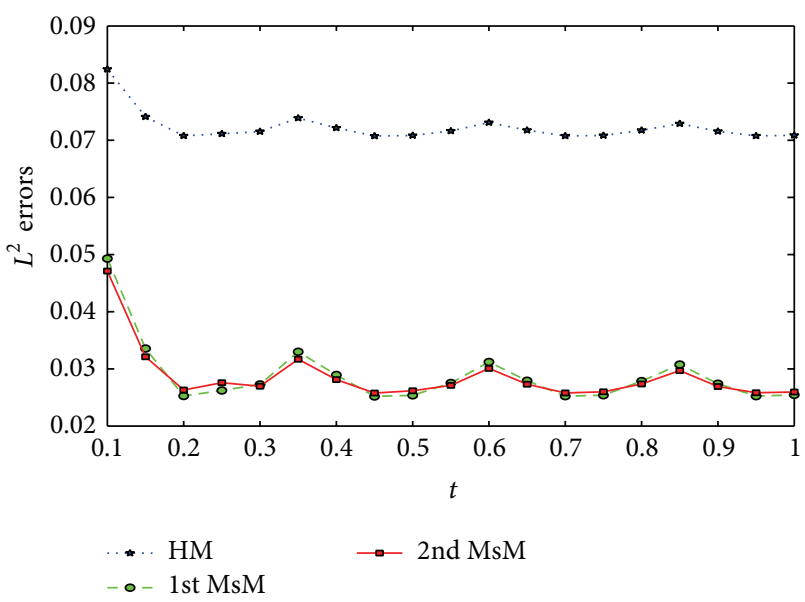

(b)

FIGURE 5: $L^{2}$-norm relative errors evolution over time at $\omega^{*}=100, M=100$ (a) Case 1; (b) Case 2.

TABLE 1: Computational cost for Case 1 and Case 2.

\begin{tabular}{lccc}
\hline & Original Eqs. & Cell Eqs. & Homogenized Eqs. \\
\hline Elements & 3145728 & 393216 & 393216 \\
Dof & 536769 & 68705 & 137410 \\
Time step & $\Delta t=0.001$ & & \\
\hline
\end{tabular}

This clearly demonstrates that the present algorithm is very effective and tremendous saving in computing time is achieved.

\section{Conflict of Interests}

The authors declare that they have no conflict of interests regarding the publication of this paper.

\section{Acknowledgments}

This work is supported by National Natural Science Foundation of China (Grant no. 11301329) and the Grant of the Firstclass Discipline of Universities in Shanghai.

\section{References}

[1] Y. Li and L. Nirenberg, "Estimates for elliptic systems from composite material," Communications on Pure and Applied Mathematics, vol. 56, no. 7, pp. 892-925, 2003.

[2] Y. Y. Li and M. Vogelius, "Gradient estimates for solutions to divergence form elliptic equations with discontinuous coefficients," Archive for Rational Mechanics and Analysis, vol. 153, no. 2, pp. 91-151, 2000.

[3] A. Bensoussan, J.-L. Lions, and G. Papanicolaou, Asymptotic Analysis for Periodic Structures, vol. 5 of Studies in Mathematics and Its Applications, North-Holland, Amsterdam, The Netherlands, 1978.

[4] V. V. Jikov, S. M. Kozlov, and O. A. Oleĭnik, Homogenization of Differential Operators and Integral Functionals, Springer, Berlin, Germany, 1994.
[5] D. Cioranescu and P. Donato, An Introduction to Homogenization, vol. 17 of Oxford Lecture Series in Mathematics and Its Applications, The Clarendon Press, Oxford University Press, New York, NY, USA, 1999.

[6] O. A. Oleĭnik, A. S. Shamaev, and G. A. Yosifian, Mathematical Problems in Elasticity and Homogenization, vol. 26 of Studies in Mathematics and Its Applications, North-Holland, Amsterdam, The Netherlands, 1992.

[7] W. Allegretto, L. Cao, and Y. Lin, "Multiscale asymptotic expansion for second order parabolic equations with rapidly oscillating coefficients," Discrete and Continuous Dynamical Systems. Series A, vol. 20, no. 3, pp. 543-576, 2008.

[8] S. Chen, W. E, and C.-W. Shu, "The heterogeneous multiscale method based on the discontinuous Galerkin method for hyperbolic and parabolic problems," Multiscale Modeling \& Simulation, vol. 3, no. 4, pp. 871-894, 2005.

[9] P. Ming and P. Zhang, "Analysis of the heterogeneous multiscale method for parabolic homogenization problems," Mathematics of Computation, vol. 76, no. 257, pp. 153-177, 2007.

[10] J. Douglas, Jr., D. Sheen, and J. E. Santos, "Approximation of scalar waves in the space-frequency domain," Mathematical Models and Methods in Applied Sciences, vol. 4, no. 4, pp. 509531, 1994.

[11] J. Douglas, Jr., J. E. Santos, D. Sheen, and L. S. Bennethum, "Frequency domain treatment of one-dimensional scalar waves," Mathematical Models and Methods in Applied Sciences, vol. 3, no. 2, pp. 171-194, 1993.

[12] D. Kim, J. Kim, and D. Sheen, "Absorbing boundary conditions for wave propagation in viscoelastic media," Journal of Computational and Applied Mathematics, vol. 76, no. 1-2, pp. 301-314, 1996.

[13] C.-O. Lee, J. Lee, and D. Sheen, "Frequency domain formulation of linearized Navier-Stokes equations," Computer Methods in Applied Mechanics and Engineering, vol. 187, no. 1-2, pp. 351-362, 2000.

[14] D. Sheen, I. H. Sloan, and V. Thomée, "A parallel method for time-discretization of parabolic problems based on contour integral representation and quadrature," Mathematics of Computation, vol. 69, no. 229, pp. 177-195, 2000. 
[15] D. Sheen, I. H. Sloan, and V. Thomée, "A parallel method for time discretization of parabolic equations based on Laplace transformation and quadrature," IMA Journal of Numerical Analysis, vol. 23, no. 2, pp. 269-299, 2003.

[16] E. Sánchez-Palencia, Nonhomogeneous Media and Vibration Theory, vol. 127 of Lecture Notes in Physics, Springer, Berlin, Germany, 1980.

[17] C.-O. Lee, J. Lee, D. Sheen, and Y. Yeom, "A frequencydomain parallel method for the numerical approximation of parabolic problems," Computer Methods in Applied Mechanics and Engineering, vol. 169, no. 1-2, pp. 19-29, 1999.

[18] L. C. Evans, Partial Differential Equations, vol. 19 of Graduate Studies in Mathematics, American Mathematical Society, Providence, RI, USA, 2nd edition, 2010.

[19] L.-Q. Cao and J.-Z. Cui, "Asymptotic expansions and numerical algorithms of eigenvalues and eigenfunctions of the Dirichlet problem for second order elliptic equations in perforated domains," Numerische Mathematik, vol. 96, no. 3, pp. 525-581, 2004. 


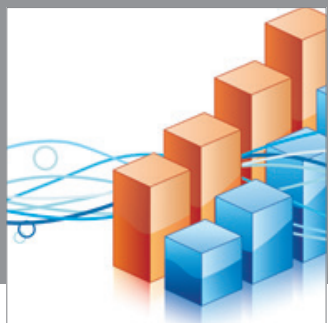

Advances in

Operations Research

mansans

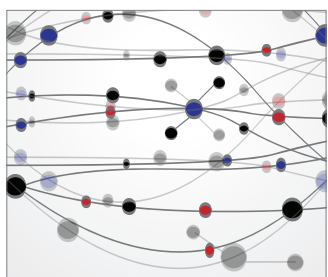

The Scientific World Journal
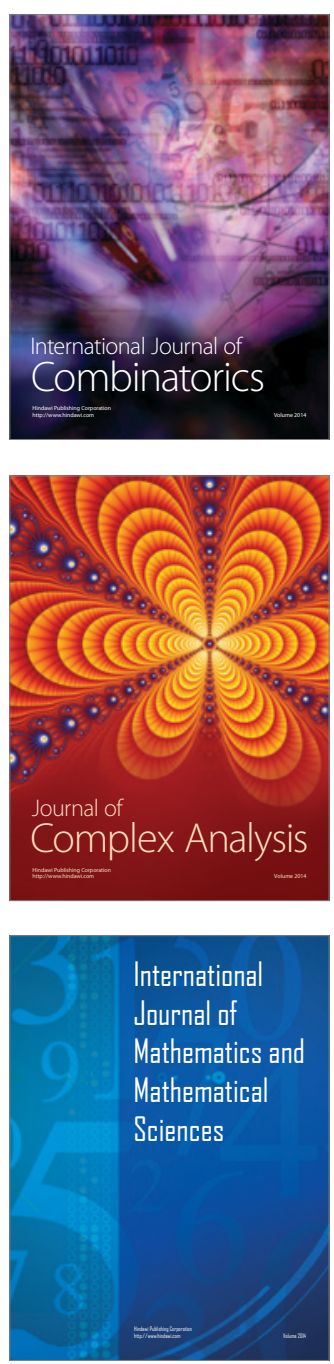
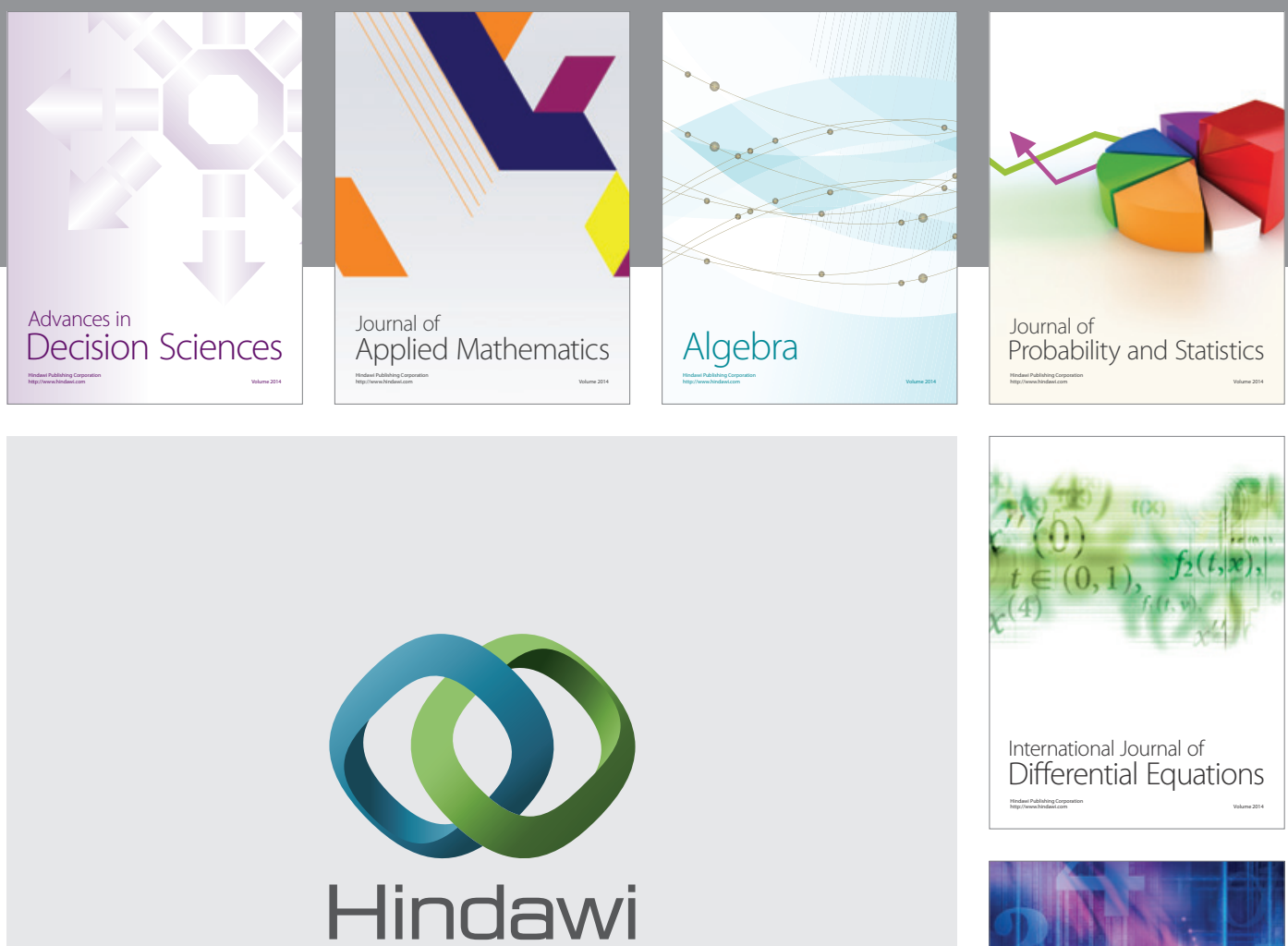

Submit your manuscripts at http://www.hindawi.com
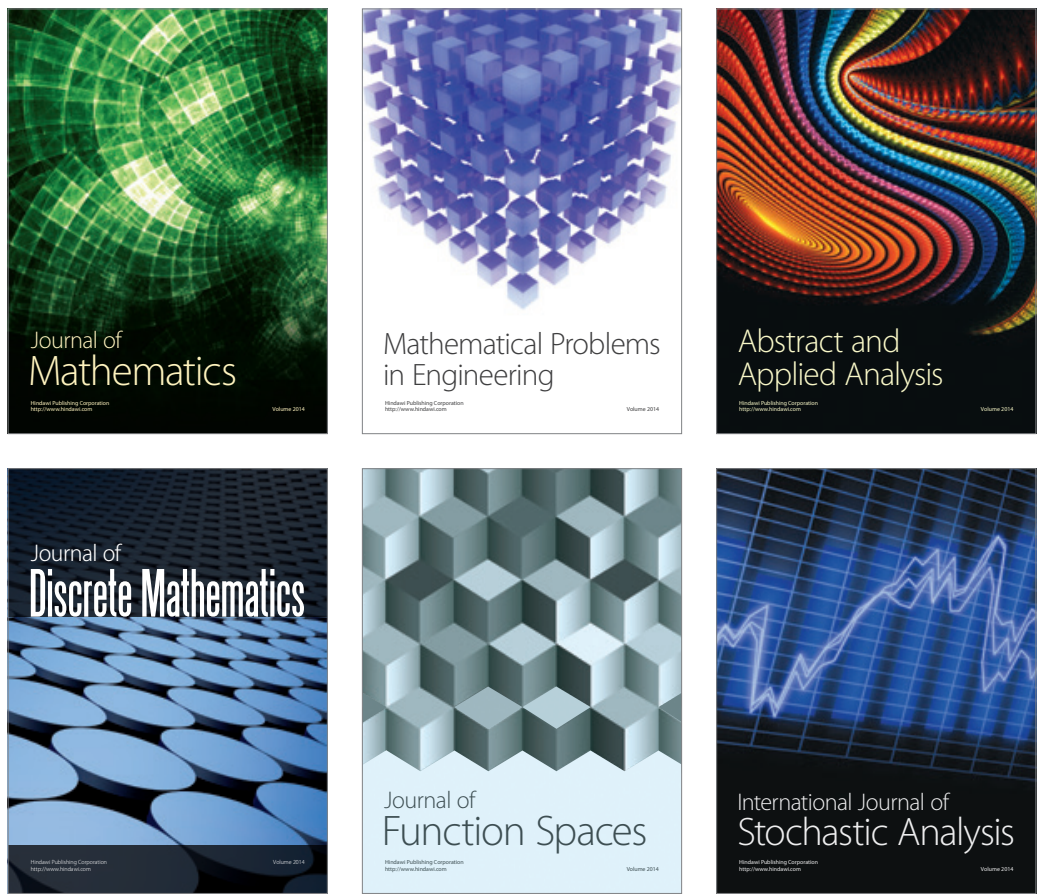

Journal of

Function Spaces

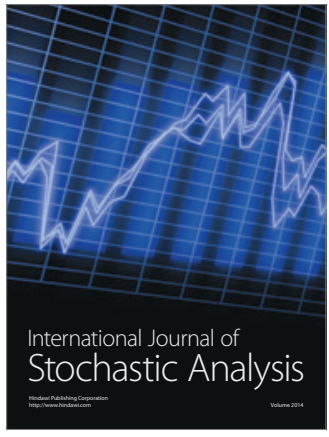

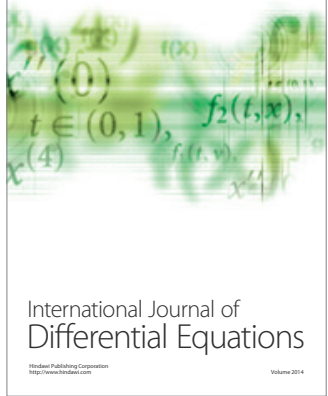
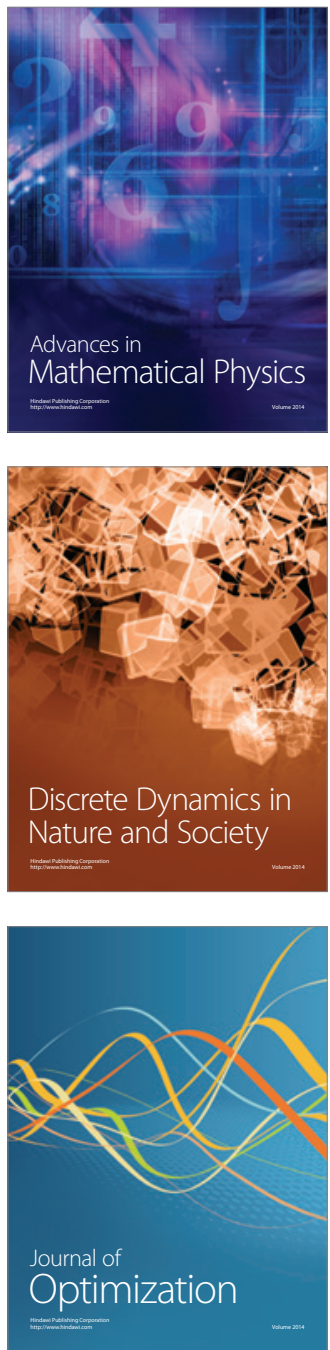